\title{
Simulation of a Piloted Turbulent Sooting Jet-Flame using a Transported-PDF and a Sectional Soot Modeling Approach
}

\author{
Tobias Dittmann* and Andreas Fiolitakis ${ }^{\dagger}$ \\ Institute of Combustion Technology, German Aerospace Centre (DLR), Stuttgart, 70569, Germany \\ Peter Gerlinger \\ IVLR, University of Stuttgart, Stuttgart, 70569, Germany \\ Manfred Aigner ${ }^{\S}$ \\ Institute of Combustion Technology, German Aerospace Centre (DLR), Stuttgart, 70569, Germany
}

In this work a Reynolds-averaged Navier-Stokes (RANS) simulation of a piloted turbulent ethylene/air jet flame is presented that combines a transported probability density function (TPDF) combustion modeling approach for the thermochemical PDF with a contemporary soot model. The soot model uses a sectional approach and a reversible model for the polycyclic aromatic hydrocarbons (PAH) that has been developed recently. Both models have been tested in earlier works successfully and they are known to have superior properties compared to simpler model approaches. In this work the TPDF and sectional soot model approaches are combined in order to predict the soot yield more accurately. Results are compared to the experiment and to an additional simulation using a standard combustion model. It is shown that the TPDF model predicts the soot volume fraction distribution better than the standard model.

\section{Introduction}

$\mathrm{T}$ HE accurate prediction of emissions that occur in technical combustion is very important when developing new combustion devices. Jet-engine designing and manufacturing companies have to deal with increasingly stricter regulations for combustion emissions like nitrogen oxides $\left(\mathrm{NO}_{x}\right)$, soot and unburned hydrocarbons (UHCs). These regulations are defined e.g. in international contracts like the Kyoto protocol from 2005, the recent Paris climate agreement and by the International Civil Aviation Organization (ICAO). Moreover, the presence of soot particles is suspected to be responsible for the formation of cirrus clouds that further reinforce the greenhouse effect [1, 2]. Therefore, it is of paramount importance to reduce combustion emissions, soot emissions in particular. The numerical simulation of combustion is a promising tool for the development of technical combustion devices that produce less soot emissions.

Modeling the formation and destruction of soot in technical combustion is very arduous since the chemical phenomena behind it are very complex and are not fully understood. There are several problems that arise in the simulation of turbulent sooting flames: First, the soot precursors, also known as polycyclic aromatic hydrocarbons or PAH in short, are known to be highly dependent on the gas phase kinetics. Second, the soot model has to be able to predict the soot yield accurately, i.e. the main formation paths and phenomena that lead to soot have to be included. Furthermore, in the presence of turbulent flows these problems are exacerbated with the closure problem of the highly non-linear formation rate of chemical species and the accurate prediction of the interaction between turbulence and chemistry.

In the present work the described problems are tackled using a transported probability density function approach in conjunction with a soot model that employs a sectional approach in both, the soot precursor model and the soot model. The flow dynamics are captured using the Reynolds averaged Navier-Stokes (RANS) ansatz. The TPDF methodology is very attractive for combustion simulations since the aforementioned closure problem of the non-linear chemical source terms is inherently avoided. The soot model employed in this work is capable to predict soot precursors accurately using

\footnotetext{
*PhD student, German Aerospace Centre (DLR), Stuttgart, 70569, Germany.

${ }^{\dagger}$ Postdoctoral Fellow, German Aerospace Centre (DLR), Stuttgart, 70569, Germany.

†Professor, IVLR University of Stuttgart, Stuttgart, 70569, Germany.

§ Professor, German Aerospace Centre (DLR), Stuttgart, 70569, Germany.
} 
a recently developed soot precursor model with reversible PAH chemistry [3]. The combination of these two approaches is promising in the accurate prediction of the soot yield.

The objective of this work is to simulate the turbulent sooting jet flame investigated by Lee et al. [4] with the aforementioned models. The flame is fueled with ethylene and is stabilized with a hydrogen pilot burner. Ethylene as a fuel seems to be a good compromise for investigating soot formation. It exhibits sufficient soot emissions and it is a simple molecule that can be described by relatively simple gas phase kinetics. In comparison, more complicated fuel, e.g. diesel or Jet A-1, requires much more species and reactions to be accurately described.

The structure of the paper is the following: First, the solver framework and the applied models are introduced. Then, the experimental as well as the computational setup of the turbulent flame is described. Afterwards, the results are presented and discussed. Finally, the work is summarized and conclusions are given.

\section{Models}

\section{A. Solver Framework}

For the computational solution of the governing equations the in-house-code THETA (Turbulent Heat Release Extension of the TAU-Code) [5, 6] is coupled with a TPDF particle solver. Since a low Mach-Number approach is applied a Poisson equation is solved for the pressure [7]. THETA is a flexible solver for multiphase, turbulent reactive flows on unstructured meshes. It is employed in this work for the solution of the conservation equations of mass and momentum together with two transport equations of turbulent variables. In this work a $k-\varepsilon$ model [8, 9] is used for turbulence closure, including the proposed round jet correction for the parameter $C_{\varepsilon 1}[10,11]$.

The conservation equations of enthalpy and species mass fractions are solved with the TPDF solver in order to handle the highly non-linear modeling closure of the chemical source terms properly. The coupling between both codes is depicted in Fig. 1. The velocity field and the turbulence variables (a turbulent time scale) are transferred to the TPDF solver. In the opposite direction averaged quantities of density and molecular viscosity, which are calculated from the particle data, are sent back to the flow solver.

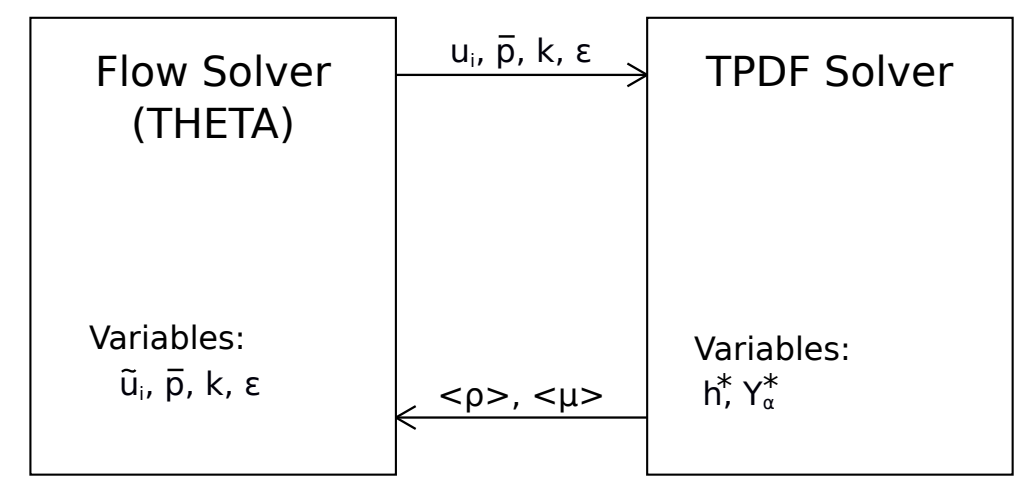

Fig. 1 Data exchange between the flow solver (THETA) and the thermochemical TPDF solver.

\section{B. TPDF Modeling}

The joint TPDF equation of the thermochemical random variables $\phi=\left[h, Y_{\alpha}\right]^{T}$ (with the corresponding composition space $\psi$ ) is solved using a Lagrangian Monte-Carlo particle solver. The TPDF equation is given by [12] (Einstein notation)

$$
\begin{aligned}
\frac{\partial \mathscr{F}_{\phi}}{\partial t}+\frac{\partial}{\partial x_{i}}\left(\left\langle u_{i} \mid \psi\right\rangle \mathscr{F}_{\phi}\right)=\frac{\partial}{\partial h}\left(\left\langle\frac{1}{\rho} \frac{\partial q_{i}}{\partial x_{i}} \mid \psi\right\rangle \mathscr{F}_{\phi}\right)+ \\
\quad \frac{\partial}{\partial Y_{\alpha}}\left(\left\langle\frac{1}{\rho} \frac{\partial j_{\alpha i}}{\partial x_{i}} \mid \psi\right\rangle \mathscr{F}_{\boldsymbol{\phi}}\right)-\frac{\partial}{\partial h}\left(\dot{q}_{r} \mathscr{F}_{\boldsymbol{\phi}}\right)-\frac{\partial}{\partial Y_{\alpha}}\left(\frac{\dot{m}_{\alpha}}{\rho} \mathscr{F}_{\boldsymbol{\phi}}\right) .
\end{aligned}
$$

$\mathscr{F}_{\phi}$ denotes the Mass Density Function (MDF) that was introduced by Pope [12], $u_{i}$ the velocity component in $i$-direction, $\rho$ the density, $\dot{q}_{r}$ the heat radiation source term and $\dot{m}_{\alpha}$ the chemical source term of the species $\alpha$. It is 
remarkable that in Eq. (1) the chemical source term appears in closed form. However, the convective and diffusive transport terms (denoted by the mathematical operator for conditional expectations, $\langle\cdot \mid \psi\rangle)$ ) require modeling.

The convective transport in Eq. (1) (second term of the left hand side) is closed by the gradient-diffusion model as proposed by Pope [12]

$$
\left\langle u_{i} \mid \psi\right\rangle \mathscr{F}_{\phi}=\tilde{u}_{i} \mathscr{F}_{\phi}-\langle\rho\rangle \Gamma_{T} \frac{\partial}{\partial x_{i}}\left(\frac{\mathscr{F}_{\phi}}{\langle\rho\rangle}\right),
$$

where $\Gamma_{T}$ is the turbulent diffusivity and $\tilde{u}_{i}$ denotes the Favre-averaged velocity component in $i$-direction calculated by the flow solver.

The closure of the diffusion terms in Eq. (11) is accomplished by employing the modified Curl model [13-15] in conjunction with a diffusion model that accounts for mean molecular diffusion transport in composition space [16]. It was found that the modified Curl model has a favorable effect on the stabilization of the flame and was preferred to simpler mixing models, e.g. the interaction by the exchange with the mean (IEM) model. The constant $\left(C_{\phi}\right)$ in the modified Curl model is set to 3.5 and the mixing frequency, denoted by $\tau_{M}$, is assumed to be proportional to the turbulent time scale and can be calculated from the turbulent kinetic energy $k$ and its dissipation rate $\varepsilon$ by

$$
\tau_{M}=\frac{1}{C_{\phi}} \frac{k}{\varepsilon} .
$$

Equation (1) can be solved by means of standard discretization methods (e.g. the finite difference method), but due to the high dimension of the composition space this would be very expensive and therefore unfeasible. Instead, as proposed by Pope [12], this equation can be solved with a Lagrangian particle methodology and reads

$$
\begin{aligned}
d X_{i}^{*} & =\left(\tilde{u}_{i}+\frac{1}{\langle\rho\rangle} \frac{\partial\left(\langle\rho\rangle \Gamma_{T}\right)}{\partial x_{i}}\right) d t+\sqrt{2 \Gamma_{T}} d W_{i}, \\
d h^{*} & =\left(\dot{q}_{r}^{*}+\tilde{V}_{h}\right) d t+\Xi_{h}^{*}\left(d t, h^{*}(t), t\right), \\
d Y_{\alpha}^{*} & =\left(\frac{\dot{m}_{\alpha}^{*}}{\rho^{*}}+\tilde{V}_{Y_{\alpha}}\right) d t+\Xi_{Y_{\alpha}}^{*}\left(d t, Y_{\alpha}^{*}(t), t\right) .
\end{aligned}
$$

$\Xi_{j}^{*}$ denotes an random change of the variable $\phi_{j}$ due to a Poisson process. Behind this process the mixing model is concealed. Particle quantities are denoted by an asterisk $\left(^{*}\right)$. The quantities $\tilde{V}_{h}$ and $\tilde{V}_{Y_{\alpha}}$ describe the mean molecular drifts in composition space [16], which are defined as

$$
\begin{gathered}
\tilde{V}_{h}=-\sum_{i=1}^{3} \frac{1}{\langle\rho\rangle} \frac{\partial\left\langle q_{i}\right\rangle}{\partial x_{i}} \\
\tilde{V}_{Y_{\alpha}}=-\sum_{i=1}^{3} \frac{1}{\langle\rho\rangle} \frac{\partial\left\langle j_{\alpha i}\right\rangle}{\partial x_{i}} .
\end{gathered}
$$

The system is solved using a Lagrangian approach. From the particle data $\left(N_{P_{j}}\right.$, the number of particles in cell $\left.j\right)$ density-weighted ensemble averages are calculated by

$$
\tilde{\phi}_{\alpha, j}=\frac{\sum_{n=1}^{N_{P, j}} m_{n}^{*} \phi_{\alpha, n}^{*}}{\sum_{n=1}^{N_{P, j}} m_{n}^{*}} .
$$

For the solution of Eq. (1) an operator splitting technique of first order of accuracy is employed that can be summarized as

$$
\mathscr{F}_{\phi}(t+\Delta t)=(I+\Delta t \mathscr{D}) \cdot(I+\Delta t \mathscr{M}) \cdot(I+\Delta t \mathscr{R}) \cdot(I+\Delta t \mathscr{T}) \cdot \mathscr{F}_{\phi}(t)+O\left(\Delta t^{2}\right) .
$$

The operator $\mathscr{D}$ denotes the diffusion step, $\mathscr{M}$ the mixing step, $\mathscr{R}$ the reaction step and $\mathscr{T}$ the transport step, respectively. $I$ denotes the identity operator. The time step $\Delta t$ is limited due to convective and diffusive transport and is calculated as in [16]. The Courant-Friedrichs-Lewy (CFL) number is set to 0.7. Using this splitting technique the TPDF equation (1) 
and respectively the system of equations (4) can be solved sequentially, i.e.

$$
\begin{aligned}
\mathscr{F}_{\boldsymbol{\phi}}^{(1)}(t) & =(I+\Delta t \mathscr{D}) \mathscr{F}_{\boldsymbol{\phi}}(t), \\
\mathscr{F}_{\boldsymbol{\phi}}^{(2)}(t) & =(I+\Delta t \mathscr{M}) \mathscr{F}_{\boldsymbol{\phi}}^{(1)}(t), \\
\mathscr{F}_{\boldsymbol{\phi}}^{(3)}(t) & =(I+\Delta t \mathscr{R}) \mathscr{F}_{\boldsymbol{\phi}}^{(2)}(t), \\
\mathscr{F}_{\boldsymbol{\phi}}(t+\Delta t) & =(I+\Delta t \mathscr{T}) \mathscr{F}_{\boldsymbol{\phi}}^{(2)}(t) .
\end{aligned}
$$

Therefore, the solution of the numerical stiff reaction step can be isolated and solved separately. The SUNDIALS CVode library [17, 18] is employed for this step. The heat radiation term in the enthalpy equation is included in the reaction step. In this work heat radiation is incorporated for the species $\mathrm{CO}_{2}, \mathrm{H}_{2} \mathrm{O}$ and soot using the optically thin medium assumption [19]. This approach models the loss of radiative power as a heat sink in the enthalpy transport equation

$$
\dot{q}_{r}=-4 \sigma_{S} \rho T^{4} \sum_{\alpha_{\mathrm{rad}}} \epsilon_{\alpha_{\mathrm{rad}}}(T) Y_{\alpha_{\mathrm{rad}}} .
$$

$\sigma_{S}$ is the Stefan-Boltzmann constant and $\alpha_{\text {rad }}$ denotes a radiating species. The species emissivity coefficients $\epsilon_{\alpha_{\mathrm{rad}}}$, which are temperature dependent, are derived from the work of Mauss [20]:

$$
\begin{aligned}
\epsilon_{\mathrm{CO}_{2}} & =\frac{46.241 \frac{1}{m} \mathcal{R}}{p_{0} M_{\mathrm{CO}_{2}}} \exp \left(-8.888 \times 10^{-4} T\right) T, \\
\epsilon_{\mathrm{H}_{2} \mathrm{O}} & =\frac{22.6 \frac{1}{m} \mathcal{R}}{p_{0} M_{\mathrm{H}_{2} \mathrm{O}}} \exp \left(-1.546 \times 10^{-3} T\right) T, \\
\epsilon_{\text {soot }} & =\frac{-3.5 \times 10^{5}}{\rho_{s}}+\frac{1735}{\rho_{s}} T,
\end{aligned}
$$

where $\mathcal{R}$ is the universal gas constant, $p_{0}$ the standard pressure ( $\left.1 \mathrm{~atm}\right), \rho_{s}$ the density of soot and $M_{\alpha}$ denotes the molecular mass of the species $\alpha$. Since heat radiation absorption effects are neglected in this model the heat radiation term in the TPDF equation (1) appears in closed form, which simplifies the turbulence-radiation-interaction (TRI) problem tremendously (see Ref. [19]).

The TPDF code is parallelized in a hybrid fashion, i.e. it combines a distributed memory approach (using the Message Passing Interface library - MPI) and a shared memory approach (using the OpenMP library). The computational domain is decomposed into sub domains and every MPI process is responsible for one sub domain. The particles are tracked over domain boundaries and its properties are communicated to the domain neighbors. To each cluster node one MPI process is assigned and every MPI process controls a team of OpenMP threads. The number of OpenMP threads coincides with the number of physical cores in the cluster node. The load balancing problem is tackled by calculating cell weights in the domain partitioning tool, which is executed prior to the simulation run. Since the most expensive part of the simulation is the reaction step, the cell weights are determined from accumulated the CPU times in chemistry solver.

\section{Chemistry and Soot Modeling}

The entire chemistry model consists of the gas phase kinetics, the PAH model and the soot model. All reactions are merged together into one large reaction mechanism that is available in CHEMKIN format [21] and is solved in the reaction step. The distinction of the gas phase, the PAHs the soot model is made according to the molecular weight as depicted in Fig. 2

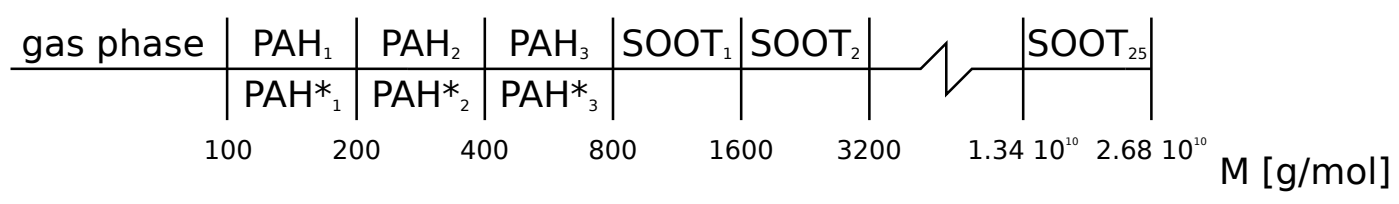

Fig. 2 The gas phase, the soot precursor and the soot model. 
The underlying chemical kinetics of the gas phase is modeled using a reaction mechanism that consists of 43 species and 304 elementary reactions [22]. This reduced mechanism has been derived from the detailed mechanism of Slavinskaya et al. [23] and has been successfully used in previous works [24-26].

The gas phase model treats aromatics up to benzene and toluene. Larger aromatics, i.e PAHs, are modeled using a sectional approach in order to reduce the computational costs. PAHs, which are defined between a molecular weight of $100 \mathrm{~g} / \mathrm{mol}$ and $800 \mathrm{~g} / \mathrm{mol}$, are discretized in three logarithmically scaled sections, cf. Fig. 2 The PAH model contains sub models for the gas phase interaction, PAH surface reactions (growth and oxidation) and PAH growth via coagulation. The interaction with the gas phase kinetics is described by a PAH formation mechanism that conveys the aromatic species benzene and the aromatic radicals phenyl and benzyl to the first PAH bin [3]. For the PAH surface growth with acetylene $\left(\mathrm{C}_{2} \mathrm{H}_{2}\right)$ the PAH model of Eberle et al. [3] is utilized. This model contains PAH radicals $\left(\mathrm{PAH}^{*}\right)$ that accounts for the reversible nature of the hydrogen-abstraction, carbon-addition (HACA) mechanism. Rate parameters are derived from a detailed reaction mechanism. Hydrogenation of PAH bins is considered by $\mathrm{H}$-atoms, O-atoms, hydroxyl (OH) and unimolecular decomposition. Further, interactions between the PAH radicals and between PAH radicals and PAH molecules are incorporated by the extended PAH coagulation sub model. More information about the employed PAH model can be found in Eberle et al. [3].

Coagulation involving $\mathrm{PAH}_{3}$ or $\mathrm{PAH}_{3}^{*}$ bins as reactants leads to the nucleation of soot. The first soot bin is defined starting with a molecular weight of $800 \mathrm{~g} / \mathrm{mol}$ that corresponds to an incipient soot diameter of $1.28 \mathrm{~nm}$. A constant soot density of $\rho_{s}=1800 \mathrm{~kg} / \mathrm{m}^{3}[27,28]$ is assumed. The soot model also utilizes the sectional approach and discretizes soot particles in 25 soot bins with logarithmically increasing molecular weights. This soot model was implemented by Blacha et al. [24] and further improved by Eberle et al. [26]. It contains sub models for soot nucleation, soot surface reactions (growth and oxidation), as well as models for PAH-soot condensation, soot coagulation and soot agglomeration. A detailed description of the soot model can be found in Eberle et al. [26].

\section{Experimental and Computational Setup}

\section{A. Burner Presentation}

The turbulent flame considered in this work is an atmospheric flame investigated by Lee et al. [4]. The flame is fueled with a jet of pure ethylene and is stabilized and attached by a pilot burner, which is operated at a low hydrogen mass flow compared to the main burner. The pilot burner basically consists of a flame holder with three rings of drilled holes, as can be seen in photo in Fig. 3 3 . The geometry of the burner is sketched in Fig. 33. The inner diameter $\left(d_{n}\right)$ of the fuel tube is $2.18 \mathrm{~mm}$. The pilot burner is enclosed between the fuel tube ( $3.96 \mathrm{~mm}$ outer diameter, o.d.) and an outer tube ( $15.06 \mathrm{~mm}$ inner diameter, i.d.). The recess depth of the flameholder amounts to $4 \mathrm{~mm}$.

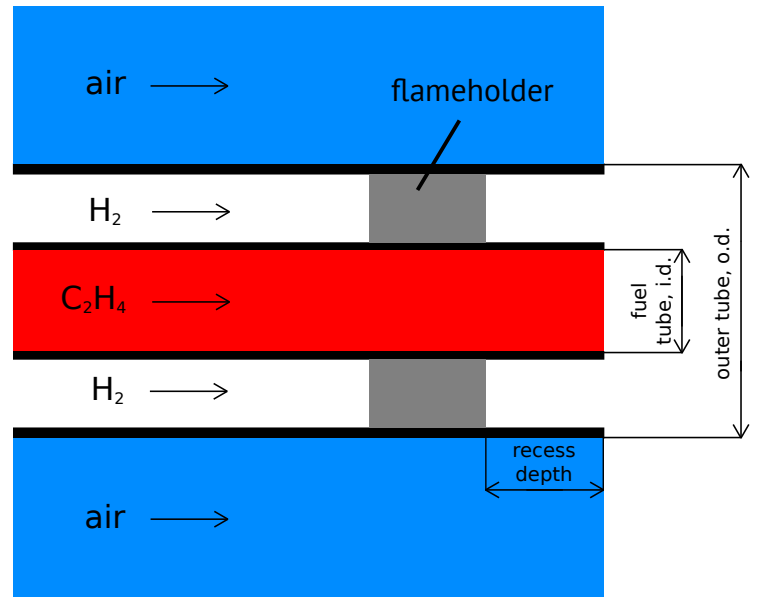

(a) The burner geometry

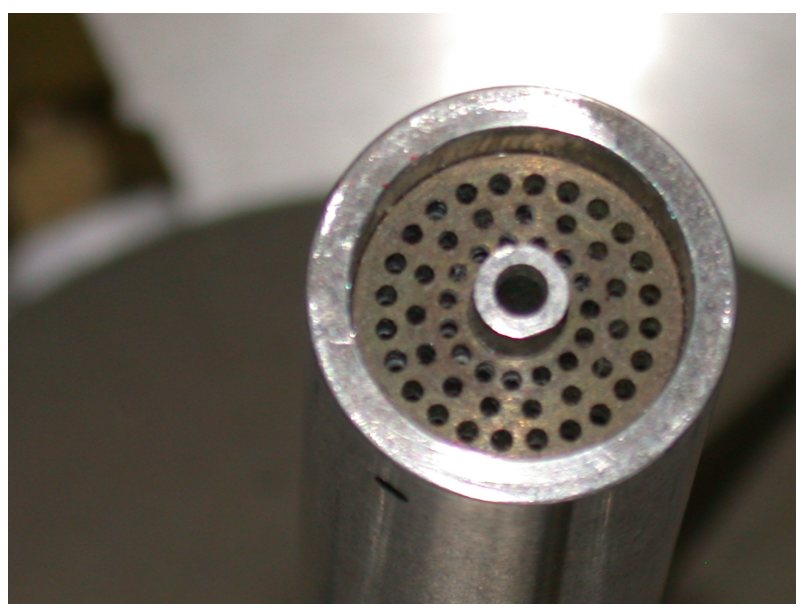

(b) A photo of the burner

Fig. 3 Illustration of the burner setup: Schematic sketch and a photo of the burner (private communication with Robert J. Santoro).

The following measurements were conducted by Lee and its coworkers [4]: Temperature by using coherent 
anti-Stokes Raman spectroscopy (CARS), measurements of the hydroxyl radical (OH) using laser-induced fluorescence (LIF, only qualitative) and the laser-induced incandescence (LII) technique was employed for measurements of the soot volume fraction.

\section{B. Computational Setup}

The Reynolds number of the jet (based on the inner tube diameter, $d_{n}$ ) is 12,000 and the mass flow rate of ethylene is $0.209 \mathrm{~g} / \mathrm{s}$. The burned hydrogen of the piloted flame is prescribed at an equilibrium state with an equivalence ratio of one. The entire mass flow rate of the pilot burner amounts to $0.0016 \mathrm{~g} / \mathrm{s}$ (based on a hydrogen mass flow rate that is $1 \%$ of the ethylene mass flow). The temperature of the burned pilot gases is calculated to be $2380.5 \mathrm{~K}$. In order to reduce the computational costs only an eight degree section of the axisymmetric configuration is simulated with an overall number of 173,650 volumes. The number of particles per cell is set to 60 and a split/annihilation algorithm ensures that this number remains approximately constant during the simulation. For RANS simulations an additional time averaging is performed in order to reduce the stochastic noise.

\section{Results}

In order to give a first impression of the flame the calculated temperature distribution is shown in Fig. 4a. The high temperatures of the pilot burner gases are clearly visible in this contour plot. The distribution of the soot volume fraction is shown in Fig. 4p. The maximal temperature of the main flame amounts to $1904 \mathrm{~K}$ and the maximal soot volume fraction is $1.353 \mathrm{ppm}$. The presence of hot, burned gases in the pilot flame keeps the ethylene flame attached to the burner. Also visible is the contraction of the flame at approximately $z / d_{n}=10$. This contraction arises from two facts. First, at the location of the contraction the ethylene jet is initially ignited from the hot pilot gases and second, the surrounding co-flowing air is rapidly soaked into the flame. This causes the flow field of the pilot flame to be bent towards the main axis. This behavior is characteristic for piloted jet flames. The soot volume fraction peaks at around 120 fuel tube diameters above the burner exit. As mentioned before the soot model incorporates oxidation due to $\mathrm{OH}$ and $\mathrm{O}_{2}$. The presence of $\mathrm{OH}$ and $\mathrm{O}_{2}$ as well as the high temperatures in the flame wings are responsible for high progress rates of soot oxidation in these areas. Therefore, the soot yield is confined at an area close to the symmerty axis, which is recognizable in Fig.4p.

In the following experimental results are compared to the simulation results of the hybrid RANS / TPDF method and to results of an additional simulation with a finite rate chemistry (FRC) model and a laminar chemistry closure (these results are henceforth denoted as LAM). The latter simulation is conducted with the same code and uses the same gas phase, soot precursors and soot models as in the TPDF simulation.

The axial temperature profile as well as radial temperature profiles at five locations above the burner exit are depicted in Fig. 5 and Fig. 6 Red lines show the results of the TPDF model, blue lines the results of the LAM simulation and the symbols represent the results of the experiment (closed symbols for the mean, open symbols for the standard deviation). Both simulation models capture the temperature distribution reasonably well. The peak temperatures in the first two radial profiles are predicted more precisely by the TPDF model. The LAM model clearly overpredicts the temperatures in this region. Also, in case of the TPDF model, the profiles of the temperature standard deviation match very well with the experiment. Unfortunately, the width of the flame is overpredicted up to a location of $z / d_{n}=60$. Starting from this location the width is captured reasonable well, but the temperature gradients at the flame wings are too high. Since the problem occurs in both, the TPDF and the LAM model this could be explained by deficiencies of the turbulence model. Moreover, the temperatures at the centerline are too low in comparison with the experiment. This circumstance has been recognized before [29]. One explanation for this issue could be the limitation of the heat radiation model. As mentioned before, the employed radiation model neglects absorption of emitted energy. This radiation absorption peaks at the centerline of the flame and could explain the low temperatures.

The profiles of the soot volume fraction $\left(f_{v}\right)$ are shown in Fig. 7 The maximal soot volume fraction at the centerline is predicted very well by the TPDF model. The standard deviation is underpredicted by a factor of three. The locations of the maximal soot fraction as well as its standard deviation is also predicted very well by the TPDF model. The LAM model underpredicts the maximal soot volume fraction by a factor of 1.6. The difference between the results of the TPDF and the LAM models seems to be due to the different modeling approaches for the turbulence-chemistry-interaction (TCI). In both simulations the production of soot starts too early. In the case of the TPDF simulation this could be ex- 


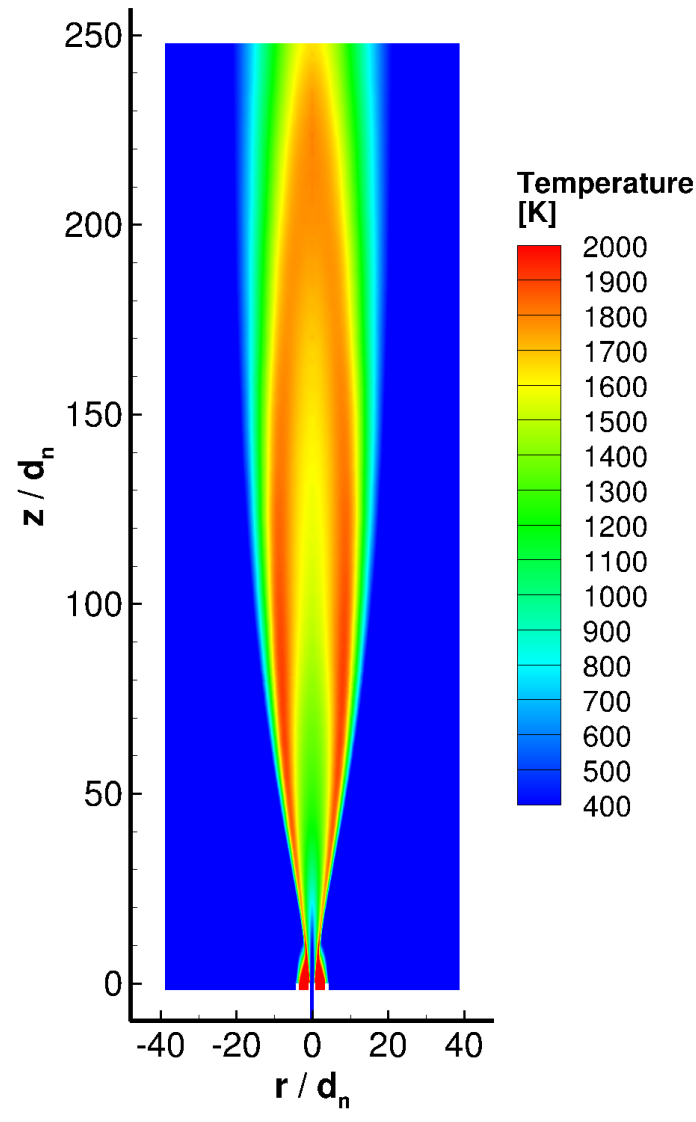

(a) The temperature distribution

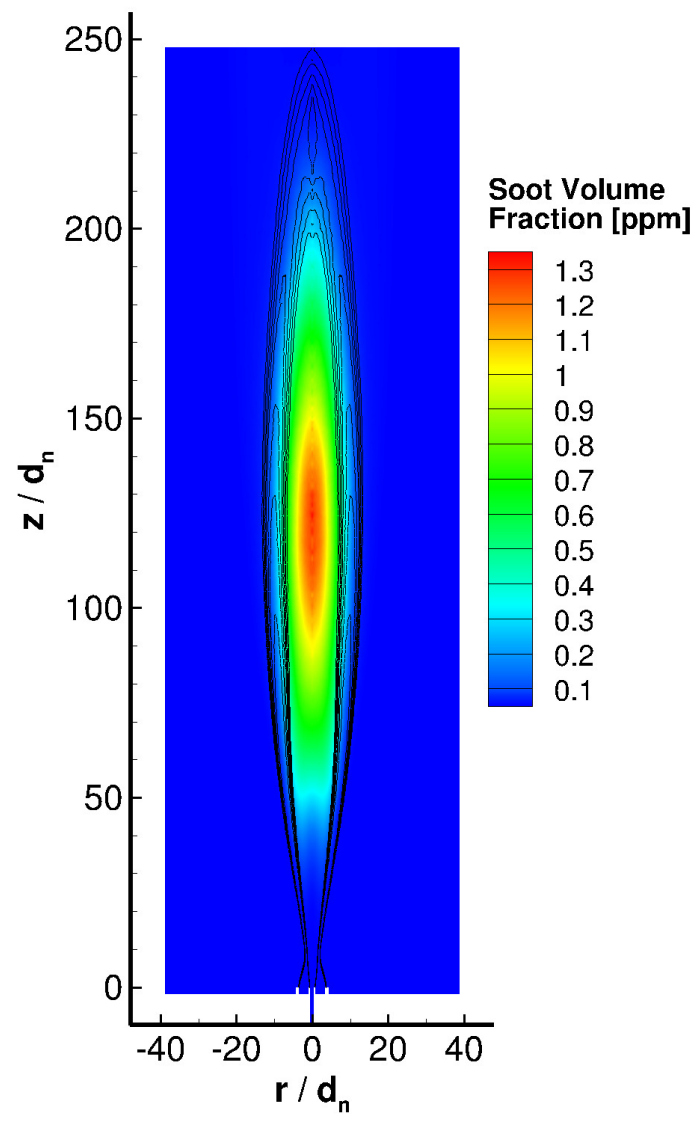

(b) The soot volume fractio distribution

Fig. 4 Temperature and soot volume fraction distributions of the hybrid RANS / TPDF simulation. Additional iso lines of the $\mathrm{OH}$ species are shown in the soot volume fraction contour plot. The results are mirrored at the z-axis.
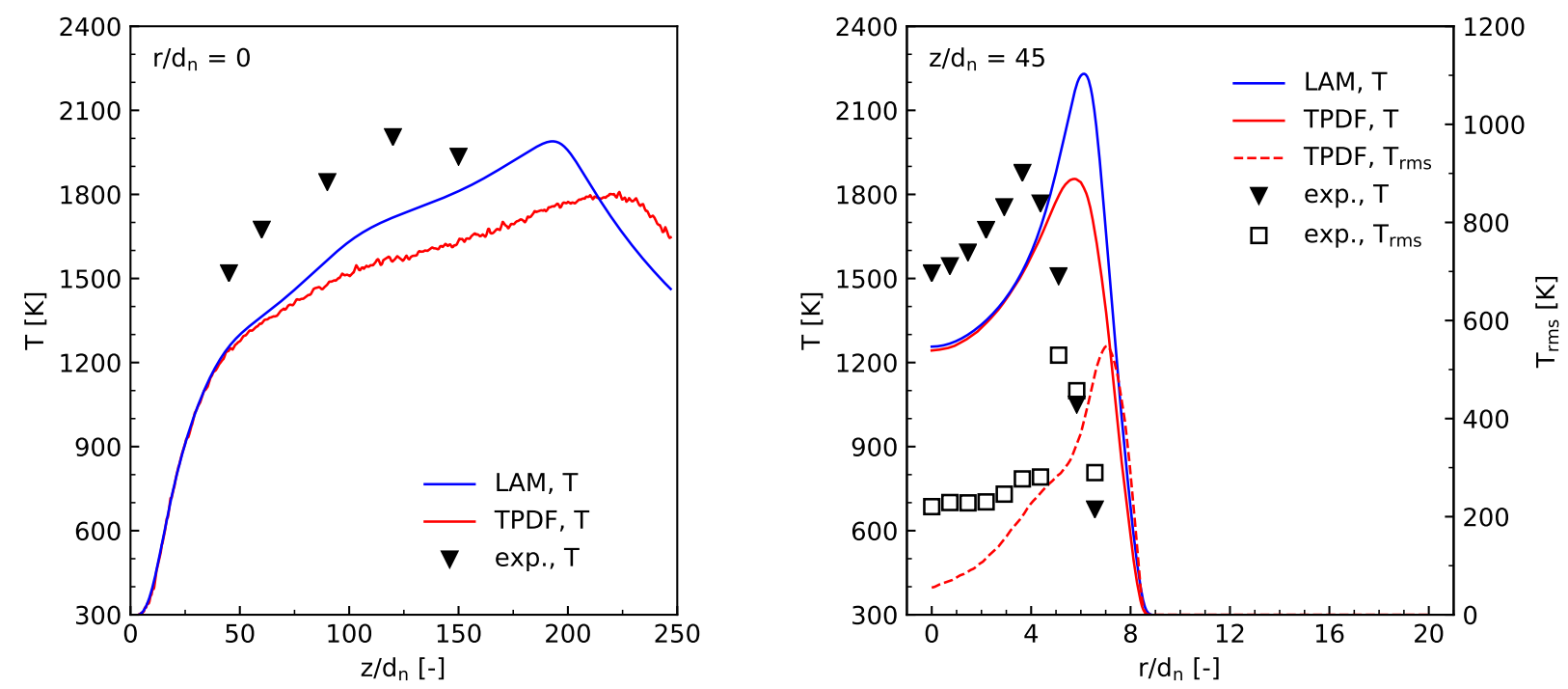

Fig. 5 Axial temperature profile and radial temperature profile at $z=34 \cdot d_{n}$. Experimental results are compared to results from LAM and TPDF simulations. 

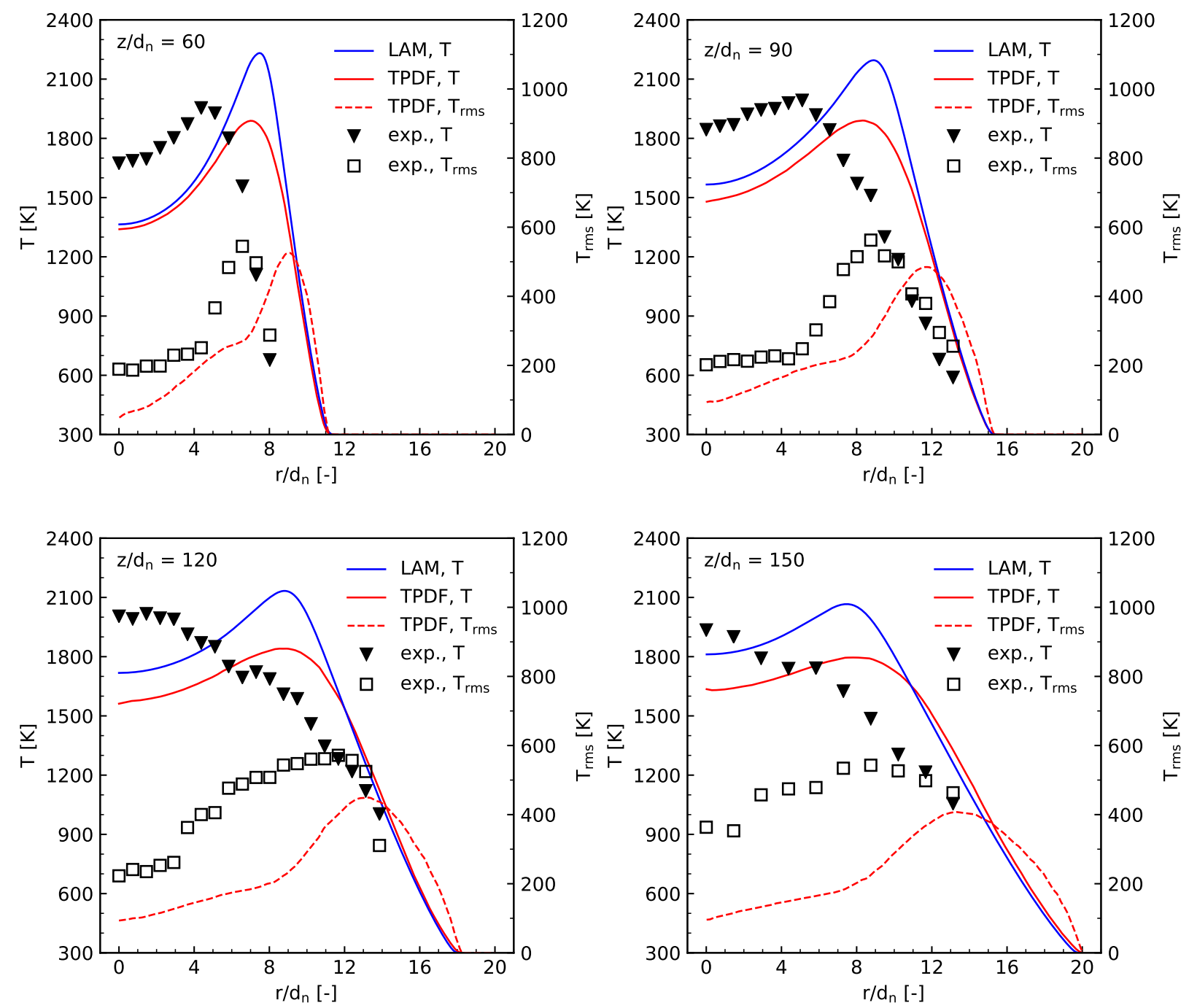

Fig. 6 Radial temperature profiles at different heights above the burner. Experimental results are compared to results from LAM and TPDF simulations.

plained by early, enhanced mixing due to the increased mixing model constant. The TPDF model also shows better results in the radial profiles of soot volume fraction. In case of the TPDF model more soot is transported in radial direction compared to the results of the LAM model. As it will be shown later, in the case of the TPDF model the soot oxidation occurs in a larger zone with smaller intensity, whereas the LAM model predicts a narrow band of soot oxidation with higher oxidation rates. It is interesting that the experiment shows even more soot transport in radial direction. This could be explained by large turbulent eddies in the shear layer of the flame that transport soot in the co-flowing air, which cannot be captured by the RANS approach.

In order to investigate the behavior of the soot formation / destruction of the sub models the soot source terms are unveiled in the following. In Fig. 8 the temperature, the soot volume fraction, the sum of all soot source terms and the soot nucleation sources of the TPDF and the LAM simulations are compared next to each other. Moreover, Fig. 9 shows the soot sources from growth by acetylene $\left(\mathrm{C}_{2} \mathrm{H}_{2}\right)$, condensation of PAH molecules as well as oxidation by $\mathrm{OH}$ and $\mathrm{O}_{2}$. In Table 1 the minimal and maximal values of the soot sub models are summarized. First of all, soot nucleation is elevated by a factor of about 1.8 in the TPDF simulation compared to the LAM model. This is due to an increased $\mathrm{PAH}_{3}$ 

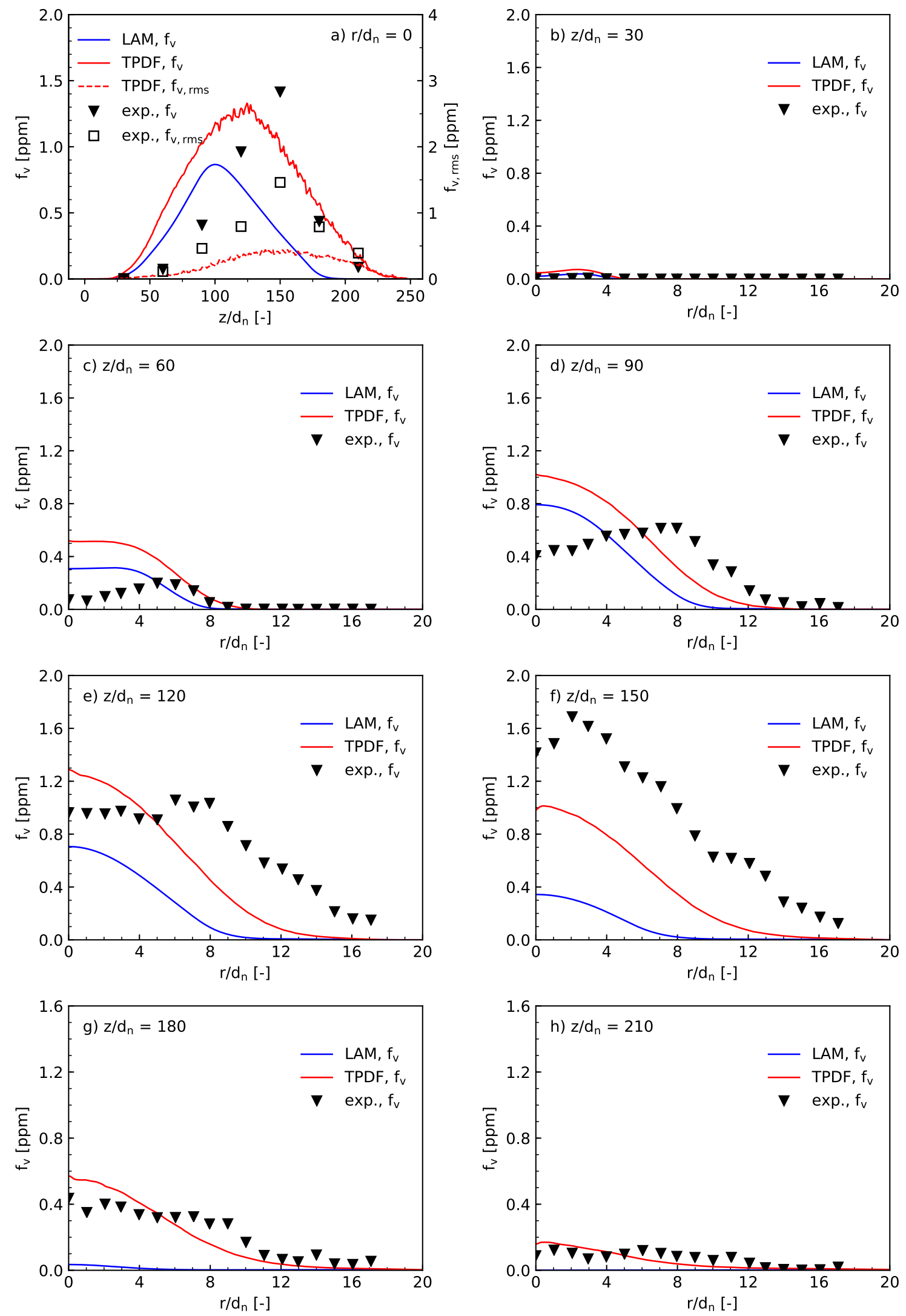

Fig. 7 Axial and radial profiles of the soot volume fraction. Experimental results are compared to results from LAM and TPDF simulations. 


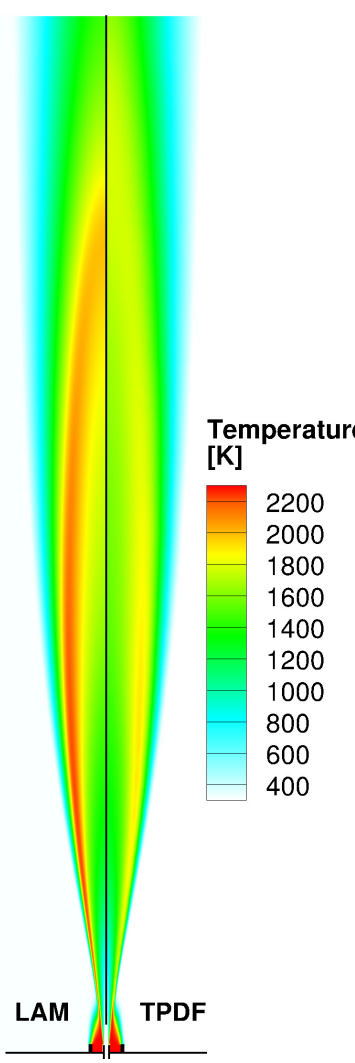

(a)

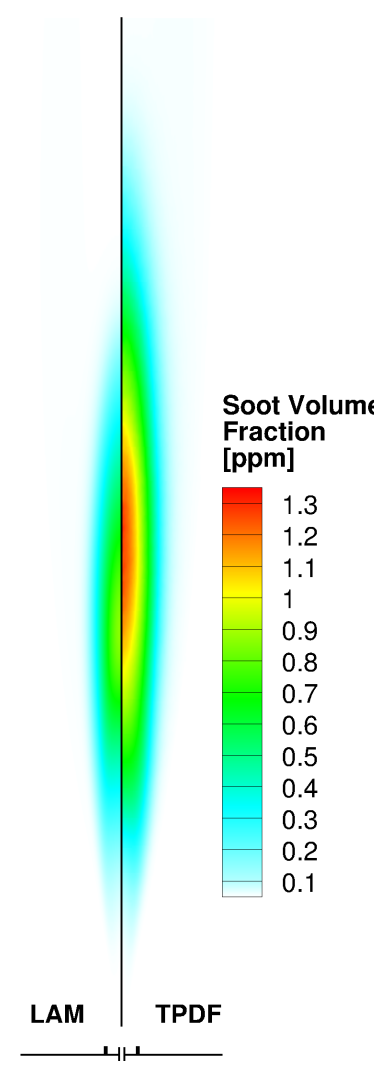

(b)

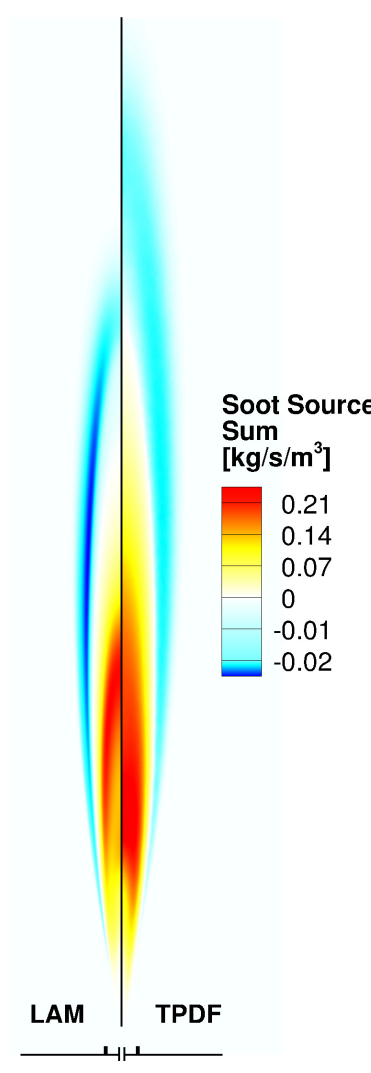

(c)

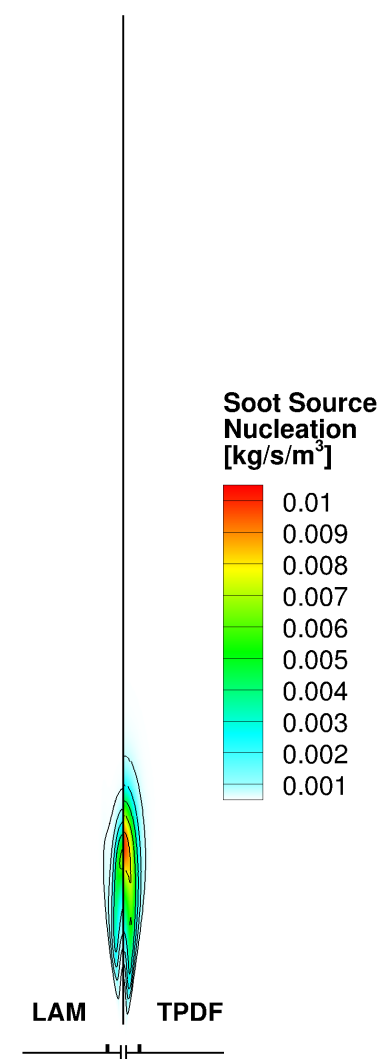

(d)

Fig. 8 Comparison of results between LAM and TPDF. a) Temperature, b) Soot volume fraction, c) Accumulated soot sources, d) Soot Nucleation, additional iso lines of third PAH species from $2 \cdot 10^{-6}$ to $1.8 \cdot 10^{-5}$.

concentration in case of the TPDF model (TPDF: $2.005 \cdot 10^{-5}$, LAM: $1.882 \cdot 10^{-5}$ ). Next, the growth of soot due to the condensation of PAH molecules onto the soot surfaces is almost twice as high in the case of the TPDF model, whereas the maximum values of accumulated PAH mass fractions in both modeling approaches are almost the same (LAM: 0.0652, TPDF: 0.0583). Soot production due to growth by $\mathrm{C}_{2} \mathrm{H}_{2}$ is greater in case of the LAM model. This arises from the fact that the maximal mass fraction of $\mathrm{C}_{2} \mathrm{H}_{2}$ is greater in the LAM simulation as well (LAM: 0.0652, TPDF: 0.0583). Soot oxidation by oxygen is similar in both simulation, albeit in case of the LAM simulation the maximal oxidation rate occurs further upstream. Finally, the oxidation of soot by $\mathrm{OH}$ is almost two orders of magnitude greater than oxidation by $\mathrm{O}_{2}$ (cf. Table 1 ) and the oxidation rate due to $\mathrm{OH}$ is six times greater in the TPDF simulation compared to the LAM simulation. As can be seen the soot sources and sinks are tightly coupled to the gas phase. Therefore, the combustion model has a strong influence on the soot yield.

The sectional soot model is able to predict soot particle size distribution (PSD). In Fig. 10. and Fig. 11 the PSDs are shown at four positions on the centerline of the burner. The soot particle diameter $\left(d_{s}\right)$ of soot bin $s$ is calculated from the mass fraction of the respective soot bin and its molecular weight (more information can be found in [26]).

Table 1 Minimal / maximal values of selected soot properties.

\begin{tabular}{lllllll}
\hline Min./max. & $\begin{array}{l}\text { Soot volume } \\
\text { fraction }[\mathrm{ppm}]\end{array}$ & $\begin{array}{l}\text { Soot Nucleation } \\
{\left[\mathrm{kg} / \mathrm{s} / \mathrm{m}^{3}\right]}\end{array}$ & $\begin{array}{l}\mathrm{C}_{2} \mathrm{H}_{2} \text { Growth } \\
{\left[\mathrm{kg} / \mathrm{s} / \mathrm{m}^{3}\right]}\end{array}$ & $\begin{array}{l}\text { PAHCondensa- } \\
\text { tion }\left[\mathrm{kg} / \mathrm{s} / \mathrm{m}^{3}\right]\end{array}$ & $\begin{array}{l}\text { OH Oxidation } \\
{\left[\mathrm{kg} / \mathrm{s} / \mathrm{m}^{3}\right]}\end{array}$ & $\begin{array}{l}\mathrm{O}_{2} \text { Oxidation } \\
{\left[\mathrm{kg} / \mathrm{s} / \mathrm{m}^{3}\right]}\end{array}$ \\
\hline LAM & 0.866 & 0.0064 & 0.1628 & 0.1295 & $-8.043 \cdot 10^{-3}$ & $-5.917 \cdot 10^{-4}$ \\
\hline TPDF & 1.353 & 0.0104 & 0.1307 & 0.2105 & $-4.814 \cdot 10^{-2}$ & $-5.983 \cdot 10^{-4}$ \\
\hline
\end{tabular}




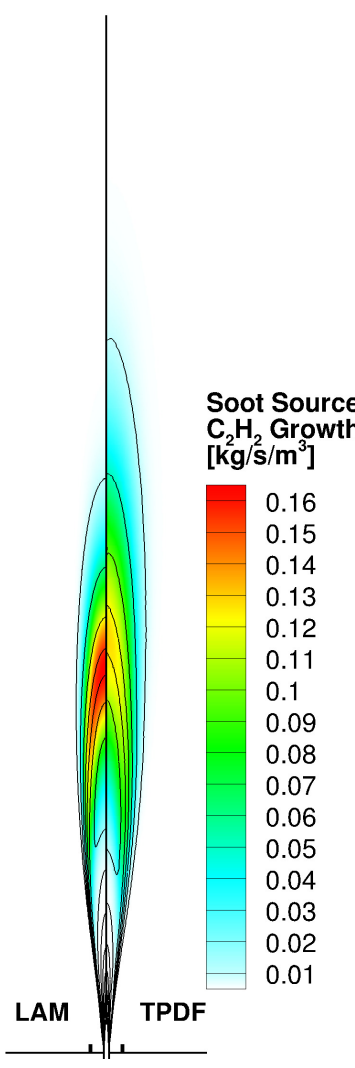

(a)

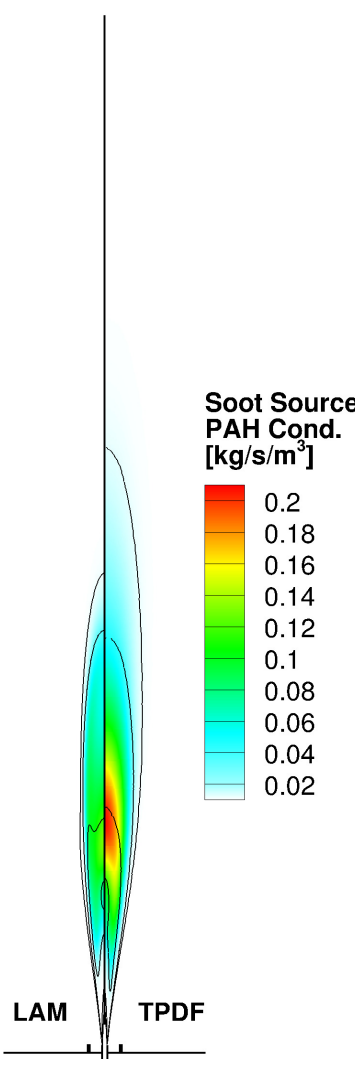

(b)

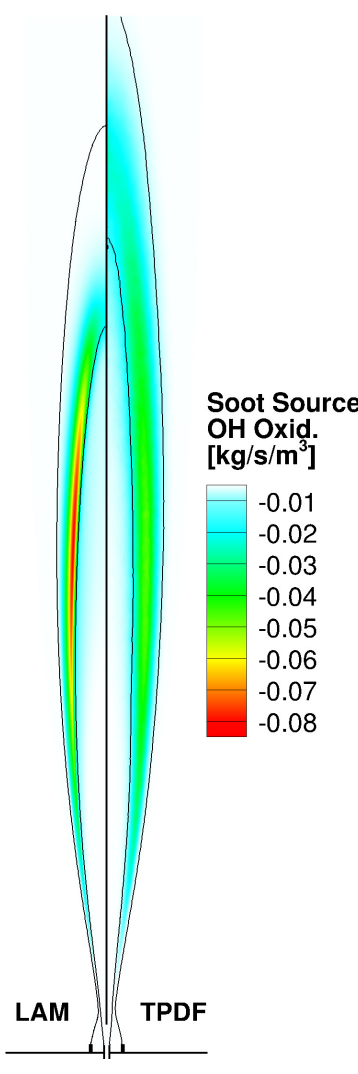

(c)

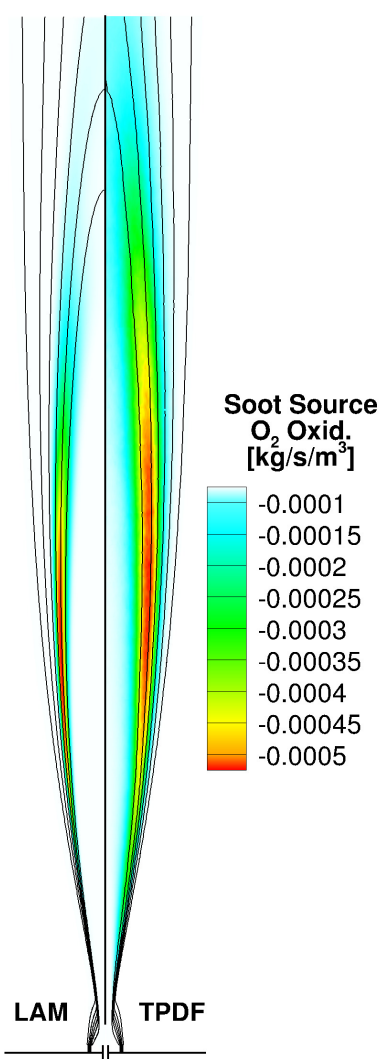

(d)

Fig. 9 Comparison of results between LAM and TPDF. a) Soot growth by $\mathrm{C}_{2} \mathrm{H}_{2}$, additional iso lines of the $\mathrm{C}_{2} \mathrm{H}_{2}$ species from 0.01 to 0.06 . b) Soot growth by $\mathrm{PAH}$ condensation, additional iso lines of accumulated PAH species at $10^{-6}, 10^{-5}$ and $10^{-4}$. c) Soot oxidation by $\mathrm{OH}$, additional iso line of the $\mathrm{OH}$ species at $2 \cdot 10^{-4}$. d) Soot oxidation by $\mathrm{O}_{2}$, additional iso lines of the $\mathrm{O}_{2}$ species from 0.02 to 0.22 .

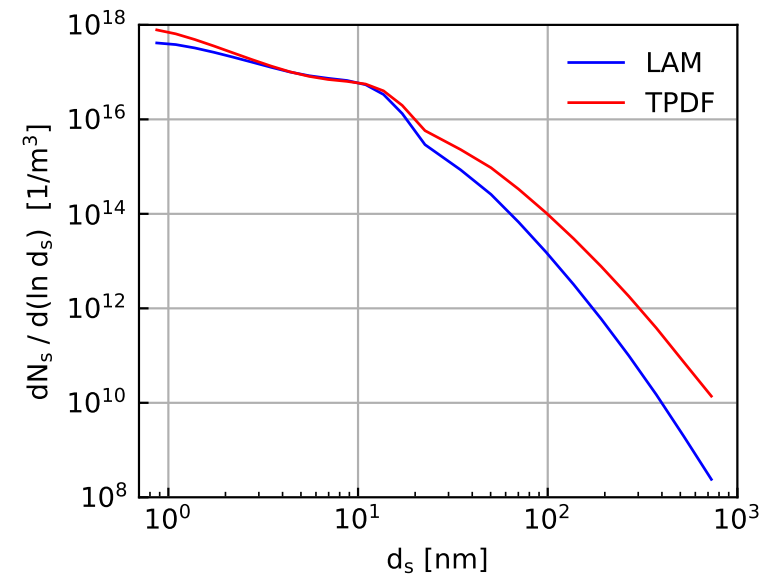

(a) PSD at $z=50 \cdot d_{n}$

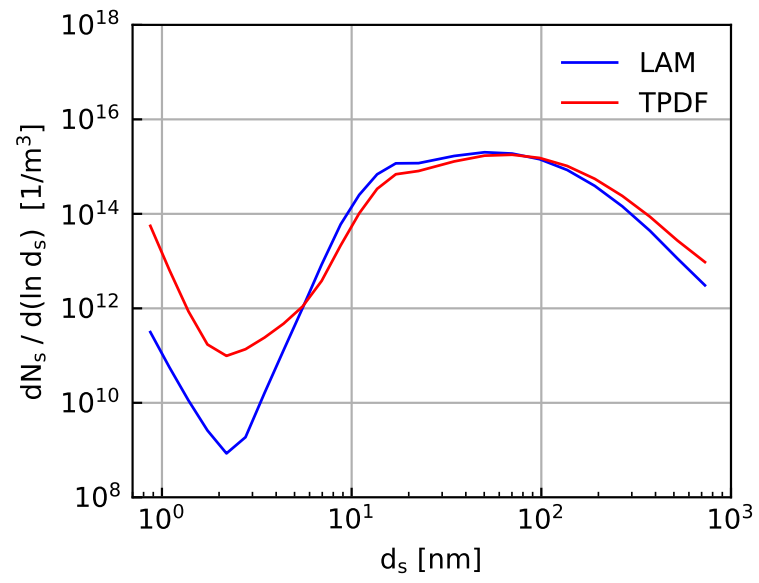

(b) PSD at $z=100 \cdot d_{n}$

Fig. 10 Particle size distributions at $z=50 \cdot d_{n}$ and $z=100 \cdot d_{n}$ (on the centerline) of the burner.

The nucleation zone is characterized by a monotonically decreasing particle size distribution and has therefore an unimodal shape. In the soot nucleation stage the TPDF simulation produces more larger particles than the LAM 
simulation. This trend is actually present in all four PSDs, especially at the farthest point to the burner exit $\left(z=200 \cdot d_{n}\right)$. At $z=100 \cdot d_{n}$ one can find a bimodal distribution and further downstream a transition takes place back to unimodal PSD shapes. The minimum in the bimodal PSD occurs at a particle diameter of about $20 \mathrm{~nm}$ in both, the LAM and TPDF simulation.

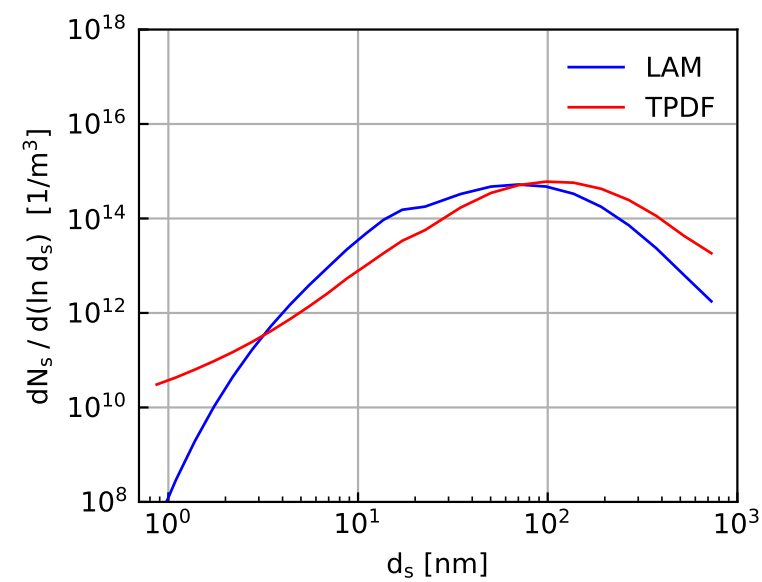

(a) PSD at $z=150 \cdot d_{n}$

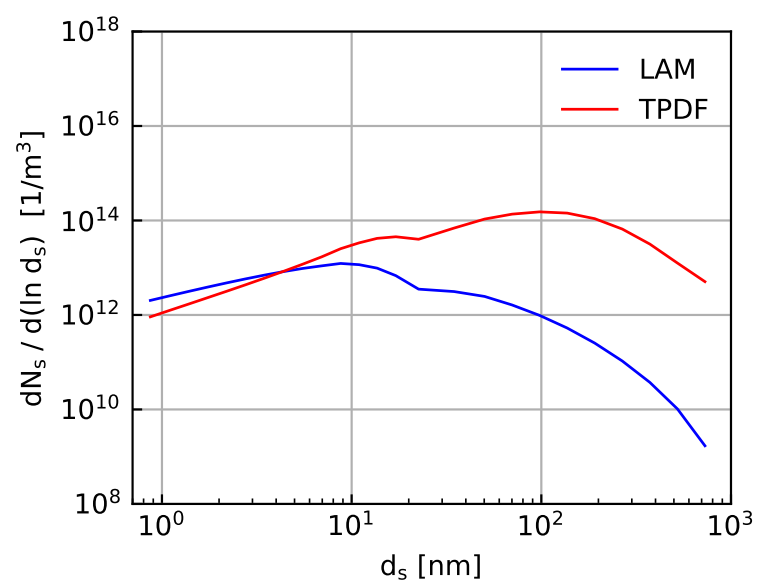

(b) PSD at $z=200 \cdot d_{n}$

Fig. 11 Particle size distributions at $z=150 \cdot d_{n}$ and $z=200 \cdot d_{n}($ on the centerline) of the burner.

\section{Summary and Conclusion}

In the present work the soot formation in a piloted turbulent ethylene jet flame is modeled with a hybrid RANS / TDPF method and a sectional model for PAHs and soot. In addition, a simulation with a laminar chemistry model is performed with the same gas phase, soot precursor and soot models. It is found that both models can reproduce the experimentally observed flame structure reasonable well. Both models show similar results in the temperature profiles. The TPDF simulation underpredicts the peak temperatures, whereas the LAM simulation overpredicts the temperatures in the front part of the flame. Unfortunately, the width of the temperature profiles in both simulation revealed deficiencies that are caused most probably by the turbulence model. There are clear differences in the soot production / destruction between both modeling approaches. The TPDF model shows better results in the soot volume fraction. Especially the maximal value of soot volume fraction matches very well with the experiment. The better accuracy of the TPDF simulation can only be caused by the superior properties of the TPDF model.

\section{References}

[1] Jensen, E. J., and Toon, O. B., "The potential impact of soot particles from aircraft exhaust on cirrus clouds," Nucleation and Atmospheric Aerosols, 1996, pp. 848-851. doi:10.1016/b978-008042030-1/50207-9.

[2] Kulkarni, G., China, S., Liu, S., Nandasiri, M., Sharma, N., Wilson, J., Aiken, A. C., Chand, D., Laskin, A., Mazzoleni, C., Pekour, M., Shilling, J., Shutthanandan, V., Zelenyuk, A., and Zaveri, R. A., "Ice nucleation activity of diesel soot particles at Cirrus relevant temperature conditions: Effects of hydration, secondary organics coating, soot morphology, and coagulation," Geophysical Research Letters, Vol. 43, 2016, pp. 3580-3588. doi:10.1002/2016g1068707.

[3] Eberle, C., Gerlinger, P., and Aigner, M., "A sectional PAH model with reversible PAH chemistry for CFD soot simulations," Combustion and Flame, Vol. 179, 2017, pp. 63-73. doi:10.1016/j.combustflame.2017.01.019.

[4] Lee, S.-Y., Turns, S. R., and Santoro, R. J., "Measurements of soot, OH, and PAH concentrations in turbulent ethylene/air jet flames," Combustion and Flame, Vol. 156, No. 12, 2009, pp. 2264 - 2275. doi:10.1016/j.combustflame.2009.09.005.

[5] Reichling, G., Noll, B., and Aigner, M., "Development of a Projection-Based Method for the Numerical Calculation of Compressible Reactive Flows," 51 st AIAA Aerospace Sciences Meeting including the New Horizons Forum and Aerospace Exposition, 2013, 2013. doi:10.2514/6.2013-1003. 
[6] Löwe, J., Probst, A., Knopp, T., and Kessler, R., "Low-Dissipation Low-Dispersion Second-Order Scheme for Unstructured Finite Volume Flow Solvers,” AIAA Journal, Vol. 54, No. 10, 2016, pp. 2961-2971. doi:10.2514/1.J054956.

[7] Oertel, H., and Prandtl, L. (eds.), Prandtl - Essentials of Fluid Mechanics, $3^{\text {rd }}$ ed., Applied Mathematical Sciences, Springer, 2010. doi:10.1007/978-1-4419-1564-1.

[8] Jones, W. P., and Launder, B. E., "The Prediction of Laminarization with a Two-Equation Model of Turbulence," International Journal of Heat and Mass Transfer, Vol. 15, 1972, pp. 301-304. doi:10.1016/0017-9310(72)90076-2.

[9] Launder, B. E., and Sharma, B. I., "Application of the Energy-Dissipation Model of Turbulence to the Calculation of Flow Near a Spinning Disk," Letters of Heat and Mass Transfer, Vol. 1, 1974, pp. 131-138. doi:10.1016/0735-1933(74)90024-4.

[10] Pope, S. B., “An Explanation of the Turbulent Round-Jet/Plane-Jet Anomaly," Journal of Computational Physics, Vol. 26, 1978, pp. 197-217. doi:10.2514/3.7521.

[11] Dally, B. B., Fletcher, D. F., and Masri, A. R., "Flow and mixing fields of turbulent bluff-body jets and flames," Combustion Theory and Modelling, Vol. 2, 1998, pp. 193-219. doi:10.1088/1364-7830/2/2/006.

[12] Pope, S. B., "PDF Methods for Turbulent Reactive Flows," Progress Energy Combustion Science, Vol. 11, 1985, pp. $119-192$. doi:10.1017/cbo9780511840531.014.

[13] Dopazo, C., "Relaxation of initial probability density functions in the turbulent convection of scalar fields," Physical Fluids, 1979, pp. 20-30. doi:10.1063/1.862431.

[14] Janicka, J., Kolbe, W., and Kollmann, W., "Closure of the Transport Equation for the Probability Density Function of Turbulent Scalar Fields," Journal of Non-Equilibrium Thermodynamics, Vol. 4, 1979, pp. 47-66. doi:10.1515/jnet.1979.4.1.47.

[15] Pope, S., "Monte Carlo PDF modelling of a turbulent natural-gas diffusion flame," Combustion Theory and Modelling, Vol. 1, 1997, pp. 79-96. doi:10.1080/713665231.

[16] Fiolitakis, A., Ess, P. R., Gerlinger, P., and Aigner, M., "Modeling of heat transfer and differential diffusion in transported PDF methods," Combustion and Flame, Vol. 161, 2014, pp. 2107-2119. doi:10.1016/j.combustflame.2014.01.021.

[17] Cohen, S. D., and Hindmarsh, A. C., "CVODE, A Stiff/Nonstiff ODE Solver in C," Computers in Physics, Vol. 10, No. 2, 1996, pp. 138-143. doi:10.1063/1.4822377.

[18] Hindmarsh, A. C., Brown, P. N., Grant, K. E., Lee, S. L., Serban, R., Shumaker, D. E., and Woodward, C. S., "SUNDIALS: Suite of nonlinear and differential/algebraic equation solvers," ACM Transactions on Mathematical Software (TOMS), Vol. 31, No. 3, 2005, pp. 363-396. doi:10.1145/1089014.1089020.

[19] Coelho, P., "Numerical simulation of the interaction between turbulence and radiation in reactive flows," Progress in Energy and Combustion Science, Vol. 33, No. 4, 2007, pp. 311-383. doi:10.1016/j.pecs.2006.11.002.

[20] Mauß, F., "Entwicklung eines kinetischen Modells der Rußbildung mit schneller Polymerisation; 1. Aufl.” Ph.D. thesis, Göttingen, 1998. URL http://publications.rwth-aachen.de/record/55085 zugl.: Aachen, Techn. Hochsch., Diss., 1997.

[21] Reaction Design, Theory Manual, Chemkin Software, 2013.

[22] Slavinskaya, N. A., and Haidn, O. J., "Reduced chemical model for high pressure methane combustion with PAH formation," Proceedings of the 46th AIAA Aerospace Sciences Meeting, AIAA, 2008. doi:10.2514/6.2008-1012.

[23] Slavinskaya, N. A., and Frank, P., "A modelling study of aromatic soot precursors formation in laminar methane and ethene flames," Combustion and Flame, Vol. 156, 2009, pp. 1705-1722. doi:10.1016/j.combustflame.2009.04.013.

[24] Blacha, T., Di Domenico, M., Gerlinger, P., and Aigner, M., "Soot predictions in premixed and non-premixed laminar flames using a sectional approach for PAHs and soot," Combustion and Flame, Vol. 159, No. 1, 2012, pp. 181-193. doi:10.1016/j.combustflame.2011.07.006.

[25] Köhler, M., Geigle, K.-P., Blacha, T., Gerlinger, P., and Meier, W., "Experimental characterization and numerical simulation of a sooting lifted turbulent jet diffusion flame," Combustion and Flame, Vol. 159, No. 8, 2012, pp. 2620-2635. doi: 10.1016/j.combustflame.2012.01.015. 
[26] Eberle, C., Gerlinger, P. M., and Aigner, M., "Large Eddy Simulations of a Sooting Lifted Turbulent Jet-Flame," 55th AIAA Aerospace Sciences Meeting, American Institute of Aeronautics and Astronautics, AIAA, 2017, p. 1785. doi: 10.2514/6.2017-1785.

[27] Donnet, J. B., Bansal, R. C., and Wang, M. J., Carbon Black, $2^{\text {nd }}$ ed., Marcel Dekker Inc., New York, 1993. doi: $10.1201 / 9781315138763$.

[28] Richter, H., Granata, S., Green, W. H., and Howard, J. B., "Detailed modeling of PAH and soot formation in a laminar premixed benzene/oxygen/argon low-pressure flame," Proceedings of the Combustion Institute, Vol. 30, 2005, pp. 1397-1405. doi:10.1016/j.proci.2004.08.088.

[29] Consalvi, J.-L., Nmira, F., and Burot, D., "Simulations of sooting turbulent jet flames using a hybrid flamelet/stochastic Eulerian field method," Combustion Theory and Modelling, Vol. 20, No. 2, 2016, pp. 221-257. doi:10.1080/13647830.2015.1125024. 